\section{EVALUACIÓN DEL}

\section{IMPACTO DE TSUNAMIS}

EN EL LITORAL

\section{PACIFICO COLOMBIANO.}

PARTE I

\section{(REGIÓN DE TUMACO)}

\section{A. QUICENO' Y M. ORTIZ ${ }^{2}$}

1 Centro Control Contaminación del Pacífico. Tumaco, Nariño, Colombia ${ }_{2}^{2}$ Departamento de Oceanografia, CICESE. Ensenada, B.C., México

\section{ABSTRACT}

The vulnerability of Tumaco region due to the impact of tsunamis generated along the Pacific coast of Colombia was analyzed. Tsunami run-up in Tumaco was evaluated by means of the numerical simulation of tsunamis generated by seismic events, similar to the December 12, 1979 Colombian earthquake; at different locations along the coast under high and low tidal conditions. The results of the numerical simulation, and the experience of the 1906 and 1979 tsunamis, corroborate the potencial tsunami risk of the region and the need of undertaking actions to mitigate material damages and loss of lives before the imminent attack of the next tsunami in the Bay of tumaco. Tsunami records of the 1979 tsunami at Buenaventura - Colombia and Esmeraldas - Ecuador, as well as the description of the 1979 tsunami effect in Tumaco were used to qualify the results of the numerical simulation.

\section{RESUMEN}

Se analiza la vulnerabilidad de la región de Tumaco ante el impacto de tsunamis generados en el litoral Pacífico Colombiano. La altura y extensión horizontal de inundación por tsunamis en Tumaco se evaluó mediante la simulación numérica de propagación de tsunamis generados por eventos sísmicos con características similares al sismo de Colombia del 12 de diciembre de 1979, ubicados en diferentes regiones del litoral bajo condiciones de marea alta y de marea baja. Los resultados de la simulación numérica, y la experiencia de los tsunamis de 1906 y 1979, corroboran la situación de riesgo de la región y la necesidad de emprender acciones de prevención para mitigar los daños materiales y la pérdida de vidas ante la inminente ocurrencia de un próximo tsunami frente a la Bahía de Tumaco. Los resultados de la simulación numérica se validaron con la descripción de los efectos del tsunami de 1979 en Tumaco y con las observaciones de este tsunami en los mareogramas de Buenaventura-Colombia y Esmeraldas - Ecuador.

\section{INTRODUCCIÓN}

Durante el siglo XX ocurrieron 4 sismos que afectaron destructivamente al litoral Pacífico Colombiano y Norte de Ecuador (fig 1). El primero de ellos fue el gran sismo-tsunami del 31 de enero de 1906 (Mw 8.8; Kanamori and MC Nally, 1982) con longitud de ruptura de aproximados $500 \mathrm{Km}$, desde Manta $\left(0.59^{\circ} \mathrm{S}\right)$ hasta Buenaventura $\left(3.54^{\circ} \mathrm{N}\right)$, (Kelleher, 1972). No obstante que el tsunami llegó con la marea baja, arrasó con todas las viviendas asentadas cerca de la playa en la Bahía de Tumaco. Se cree que murieron entre 500 y 1500 personas por causa del tsunami (Soloviev and Go, 1984). Posteriormente, al norte de Ecuador, y entre Ecuador y Colombia, ocurrieron los sismos del 14 de mayo de 1942 (MW > 7; Kelleher, 
and Mc Nally, 1982). En la Bahía de Tumaco y en el Golfo de Guayaquil se reportaron daños por el tsunami de 1958; un reporte de prensa menciona que la Isla Gorgona en la Bahía de Buenaventura fue inundada por una enorme ola después del sismo de 1942. Sin embargo, no se encontró evidencia de tsunami en el mareograma de Buenaventura (Soloviev and Go, 1975). La región al NE del área de ruptura de 1958 que aparentemente rompió en 1906 fue considerada acertadamente por Kelleher (1972), como una región de alto riesgo sísmico. Siete años después de las observaciones de Kelleher, y 73 años después del sismo de 1906, ocurrió el sismo-tsunami del 12 de diciembre de 1979 (MW 8.2) con longitud de ruptura estimada entre $180 \mathrm{Km}$ y $230 \mathrm{Km}$ (Kanamori and McNally, 1982; Beck and Ruff, 1984). Este sismo llenó aparentemente la brecha sísmica restante entre 1958 y 1906. De acuerdo a la descripción de Herd et al., (1981), y de Soloviev y Go (1975), el tsunami afectó a 15 aldeas de pescadores en la región costera desde Tumaco hasta Buenaventura e inundó completamente a 6 de estas aldeas. En Tumaco murieron al menos 220 personas por causa del tsunami; una embarcación desapareció con 40 pescadores abordo.

Pronosticar el siguiente sismo en la región está fuera del alcance de este estudio, cuyo objetivo a mediano plazo es el de elaborar cartas de inundación (tabla de tiempos y mapas de inundación por tsunamis) que constituyan el soporte técnico para coadyuvar en la creación de un sistema regional de alerta de tsunamis y en la planificación de un desarrollo urbano que permita mitigar los daños que sin lugar a duda, de acuerdo a los antecedentes de la región, serán ocasionados por el siguiente tsunami. Las cartas de inundación podrían elaborarse con sentido común considerando la experiencia de tsunamis regionales anteriores y la posibilidad de que un tsunami ocurriese en conjunción con la marea más alta. También podrían elaborarse mediante diagramas de refracción de ondas identificando e interpretando apropiadamente regiones de convergencia o divergencia de energía o bien, simulando la propagación de tsunamis mediante la solución numérica de las ecuaciones que describen la propagación de ondas largas en aguas someras (Goto et al., 1997). Este último método, a diferencia de los otros, permite calcular la altura del tsunami y la extensión horizontal de inundación y permite identificar algunos lugares de alto riesgo que en ocasiones se escapan al sentido común. En este estudio hemos empleado la solución numérica propuesta por Goto et al., (1997) para elaborar cartas de inundación de las partes insular y continental de la región de Tumaco, considerando diferentes escenarios sísmicos tsunamigénicos y la posibilidad de que el tsunami ocurriese en marea media y en marea alta. La marea es un factor determinante en el riesgo de inundación por tsunamis debido a que la diferencia de nivel entre la pleamar y la bajamar durante las mareas altas llega a ser mayor de $4 \mathrm{~m}$ en esta región. Aún cuando todas las poblaciones en el litoral merecen la misma atención, hemos iniciado el estudio en Tumaco por considerar que este puerto, con una población que sobrepasa los cien mil habitantes y con un relieve muy plano que limita los lugares de refugio, es una de las áreas de más alto riesgo de inundación por tsunami en la región. Este proyecto tiene contemplado continuar el análisis de riesgo de inundación por tsunamis en varias de las poblaciones del litoral colombiano.

\section{Propagación del Tsunami del 12 de diciembre de 1979}

La calidad de los resultados de la simulación numérica de propagación de tsunamis debe juzgarse en función de resultados esperados que dependen, entre otros factores, de la condición inicial del tsunami y de una buena representación digital del lecho marino.

Como caso de prueba para analizar los resultados del modelo de propagación de tsunamis se calculó la deformación vertical del lecho marino producida por un sismo con mecanismo focal similar al del sismo de Tumaco del 12 de diciembre de 1979. El mecanismo focal del sismo y los efectos del tsunami se describen en Herd et al., 1981; Beck and Ruff, 1984; Soloviev et al., 1992. La forma inicial del tsunami (fig 2) se consideró como una perturbación instantánea de la superficie del océano igual a la deformación vertical cosísmica del lecho marino calculada con el modelo de dislocación de Mansinha y Smylie, 1971. En el modelo de dislocación se consideró un mecanismo focal inverso - oblicuo con deslizamiento promedio de 4 metros en dirección $\mathrm{N} 35^{\circ} \mathrm{W}$ sobre un plano de falla de $160 \mathrm{Km}$ de largo con rumbo $\mathrm{N} 35^{\circ} \mathrm{E}$ y de $70 \mathrm{Km}$ de ancho con echado de $30^{\circ}$. El extremo SE del plano de falla se hizo coincidir con el epicentro del sismo del 12 
de diciembre de $1979\left(1.584^{\circ} \mathrm{N}, 79.386^{\circ} \mathrm{W}\right.$; Herd et al., 1981).

La propagación del tsunami se simuló con la solución numérica de las ecuaciones de aguas someras (Goto et al., 1997), permitiendo una condición de frontera móvil en la costa para evaluar la altura y extensión de la inundación en Tumaco:

$\left.\begin{array}{l}\frac{\partial \eta}{\partial t}+\frac{\partial_{U}}{\partial x}+\frac{\partial_{V}}{\partial y}=0 \\ \frac{\partial_{U}}{\partial t}+\frac{\partial}{\partial x}\left(\begin{array}{l}V^{2} \\ D\end{array}\right)+\frac{\partial}{\partial y}\left(\begin{array}{l}U V \\ D\end{array}\right)+g D \frac{\partial \eta}{\partial x}+g^{D^{\prime 2}} U \cdot \overline{U^{2}+V^{2}}=0 \\ \frac{\partial_{V}}{\partial t}+\frac{\partial}{\partial x}\left(\begin{array}{l}U V \\ D\end{array}\right)+\frac{\partial}{\partial y}\left(\begin{array}{l}V^{2} \\ D\end{array}\right)+g D \frac{\partial \eta}{\partial y}+\frac{g m^{2}}{D^{7 / 3}} V \cdot U^{2}+V^{2}=0\end{array}\right\}$ (1)

En estas ecuaciones, $t$ es el tiempo, $n$ representa el desplazamiento vertical de la superficie del agua respecto al nivel de equilibrio, $g$ es la aceleración gravitacional, $h$ la profundidad media de la columna de agua, $D=(n+h)$ la profundidad instantánea de la columna de agua. $U$ y $V$ son los gastos por unidad de área lateral en las direcciones longitudinal $(x)$ y latitudinal (y) respectivamente, siendo $m$ el parámetro de rugosidad de Manning, considerado constante e igual a 0.025 en este estudio. La validez del conjunto de ecuaciones (1) para simular la propagación de ondas barotrópicas se estableció desde finales del siglo XVIII (Lamb, 1932). Desde entonces las ecuaciones (1) han sido discutidas e ilustradas por un gran número de autores ( $\mathrm{Ej}$. Pedlosky, 1979). Recientemente se han comparado con éxito los resultados de la solución numérica de las ecuaciones (1) con observaciones de tsunamis transoceánicos en mar abierto (Ortiz et al., 2000a). La propagación de tsunamis transoceánicos en batimetrías costeras muy irregulares se ha simulado con el conjunto de ecuaciones (1) y con la aproximación lineal de éstas según el caso (Ortiz et al., 2000b; Ortiz et al., 2000c). La condición de inundación (frontera móvil) en el método de Goto et al., (1997) se ha empleado con éxito para reproducir la inundación por tsunamis (Shuto et al., 1986; Takahashi et al., 1995).

Las ecuaciones (1) se integraron en un esquema de diferencias finitas centrales en un conjunto interconectado de mallas con diferente resolución espacial. Se empleó una resolución espacial de 27 segundos para describir la batimetría de la región oceánica y una resolución espacial de 3 segundos para describir la batimetría de la Bahía de Tumaco. Para describir el relieve topográfico de las islas Tumaco y
El Morro y la batimetría aledaña a las islas se empleó una resolución espacial de 1 segundo $(-30 \mathrm{~m})$ obtenida recientemente por el Centro Control de Contaminación del Pacífico. La batimetría de la región oceánica se interpoló del banco de datos ETOPO2 de Smith and Sandwell, 1997. Los resultados del modelo se obtuvieron en forma mapas de inundación y de series cronológicas de nivel del mar (mareogramas sintéticos).

La localización del plano de falla y la dirección oblicua del deslizamiento sobre el plano de falla fueron necesarias para reproducir la fase negativa observada en el mareograma de Esmeraldas - Ecuador y para reproducir el tiempo de arribo del tsunami observado tanto en el mareograma de Esmeraldas como en el mareograma de Buenaventura. En las figuras 3 y 4 se muestran respectivamente los mareogramas de Esmeraldas y de Buenaventura. Estos mareogramas son los únicos registros regionales del tsunami que estuvieron a nuestra disposición. El mareograma de Esmeraldas se obtuvo de Soloviev et al., 1992, y la sección del mareograma de Buenaventura se obtuvo de Ramírez y Goberna, 1980. En las figuras 5 y 6 se comparan las observaciones del tsunami en Esmeraldas y en Buenaventura con los correspondientes mareogramas sintéticos. Ambos mareogramas sintéticos reproducen aproximadamente las principales características del tsunami observado.

En el mareograma sintético de Esmeraldas se puede apreciar la recesión en el nivel del mar minutos después del tiempo de origen del sismo (07:59:04 GMT; 02:59:04 hora local) y posteriormente el arribo de un pulso positivo.

En el mareograma de Buenaventura es difícil identificar con precisión el inicio del tsunami debido a la calidad de la copia del mareograma y posiblemente debido a una atenuación en amplitud por la respuesta del mareógrafo. Sin embargo, si se compara el tiempo del primer máximo se aprecia que el tsunami sintético llega 5 minutos antes que el observado. Esta diferencia de 5 minutos restringe la longitud del plano de falla, por lo que para hacer coincidir el tiempo del primer máximo es necesario reducir la longitud del plano de falla de $160 \mathrm{Km}$ a $120 \mathrm{Km}$. Por otra parte, el proceso de ruptura del sismo de Tumaco de 1979 (Beck and Ruff, 1984) indica una longitud de ruptura 
del momento sísmico (80\%) ocurre en los primeros 120 $\mathrm{Km}$ a partir del epicentro. Posiblemente la dislocación cosísmica en los restantes $60 \mathrm{Km}$ generó una perturbación de escasos centímetros en el nivel del mar que no puede ser observada en el mareograma debido a la respuesta del mareógrafo.

En resumen, aún cuando se ha empleado un plano de falla sin asperezas y un modelo sencillo para calcular la forma inicial del tsunami, se puede considerar que los resultados de la propagación del tsunami son alentadores porque reproducen aceptablemente las principales características del tsunami observado en ambos extremos del plano de falla (Esmeraldas y Buenaventura).

Desafortunadamente no contamos con observaciones del tsunami en la región de interés ya que el mareógrafo de Tumaco se dañó durante el sismo. Sin embargo, los resultados de la simulación del tsunami en Tumaco pueden compararse indirectamente con la descripción de los efectos locales del tsunami. Por ejemplo, la altura del tsunami en la costa norte de Tumaco $(0.8 \mathrm{~m}$; Herd et al ., 1981) concuerda aproximadamente con la altura $(0.7 \mathrm{~m})$ del tsunami en el mareograma sintético correspondiente a la localidad \# 3 de figura 7. En la figura 8 se muestran los mareogramas sintéticos correspondientes a las localidades \#1, \#2, \#3 y \#4 indicadas en la figura 7. El primer máximo $(1.8 \mathrm{~m})$ del tsunami llega a la localidad \#4 (frente a la isla El Morro) después de 30 minutos del tiempo de origen del sismo. Paradójicamente, en la localidad \#3 el tsunami llega 9 minutos más tarde que en la localidad \#4. Este retraso ocurre debido a que el frente del tsunami se propaga por una región de muy poca profundidad $(<2 \mathrm{~m})$ en su trayectoria a la localidad \#3. Como consecuencia de la fricción y de la poca profundidad el frente del tsunami adquiere una forma muy pronunciada. Posiblemente en la situación real el tsunami llegó en forma de salto hidráulico a la costa norte de Tumaco. Los resultados de altura y extensión de inundación en las islas se muestran en la figura 9. El tsunami de 1979 ocurrío por fortuna durante la bajamar del 12 de diciembre (ver figuras 3 y 4 ). Suponiendo que el tsunami hubiese ocurrido durante la marea alta del mismo día (+1.75 m sobre el nivel medio del mar; NMM), la inundación hubiese cubierto la mayor parte de las islas y hubiese alcanzado la parte urbana en el continente (figura 10).
Tiempo de arribo y altura del tsunami en Tumaco para diferentes escenarios sismicos regionales

Es evidente que un sismo-tsunami que ocurra frente a la Bahía de Tumaco representa el mayor riesgo de inundación en Tumaco, sin embargo es importante evaluar el potencial de inundación en Tumaco debido a sismos-tsunami que ocurran a lo largo del litoral Pacífico Ecuador - Colombia. Con este objetivo se simuló la propagación de 4 tsunamis generados a lo largo del litoral desde el norte de Ecuador hasta el norte de Buenaventura. La magnitud de la dislocación cosísmica se eligió similar a la del sismo 1979, considerando un mecanismo focal puramente inverso y modificando el rumbo de la falla que en cada uno de los casos alineó paralelo a la zona de subducción. En los experimentos se mantuvo constante el nivel del mar fijándolo en el nivel de marea media. En la Figura 11 se indica la posición de cada uno de los planos de falla (a, b (1979), c, d) considerados en los experimentos. En la Tabla 1, se muestran el tiempo de arribo y la altura de cada uno de los tsunamis en las cuatro localidades de Tumaco indicadas en la Figura 7. Los resultados de los cuatro experimentos muestran que la mayor altura del tsunami ocurre en la localidad \#4 al NE de la Isla Morro. Los tsunamis generados tanto al sur como al norte del área de ruptura del sismo de 1979 no representan una situación de alto riesgo desde el punto de vista de la inundación en Tumaco. Sin embargo, la corriente asociada a una altura de tsunami de $40 \mathrm{~cm}$ puede representar un peligro considerable para la navegación costera.

\section{Discusión y Conclusiones}

La experiencia de los tsunamis de 1906 y 1979 en el litoral Pacífico Colombiano nos ha mostrado sin lugar a duda que las poblaciones costeras están expuestas al riesgo de inundación por tsunamis, especialmente si estos ocurren en conjunción con marea alta. Los mapas de inundación de Tumaco muestran consistentemente la situación de amenaza de la región ante el impacto de tsunamis generados por sismos con características similares al de 1979. No es necesario suponer un evento extremo de tsunami para constatar la situación de riesgo en Tumaco. Dadas las condiciones del terreno, un tsunami similar al de 1979 en conjunción con marea alta podría cubrir más del $80 \%$ de la localidad de Tumaco. Adicionalmente existe una serie de condiciones 
particulares que la hacen altamente vulnerable ante este tipo de fenómeno, entre las que se encuentran: la poca capacidad de soporte de sus suelos; la ausencia de normas sismorresistentes en sus construcciones; los densos asentamientos humanos en regiones expuestas directamente al embate de olas; la falta de terrenos elevados y de vías de evacuación; la falta de percepción del riesgo por parte de la comunidad y la explosión demográfica de los últimos años. En 1979 la población del municipio de Tumaco era de 80000 habitantes y en la actualidad se estima superior a 120000 habitantes.

En la Figura 12 se muestra una fotografía aérea de Tumaco con la finalidad de ilustrar la densidad demográfica actual y el consecuente incremento en la magnitud de daños potenciales por tsunami con respecto al evento de 1979 .

El análisis de tiempos de arribo de tsunami a la región de Tumaco ( 30 minutos) muestra la necesidad de contar con un sistema regional de alerta de tsunamis. Sin embargo, para que un sistema de alerta sea eficaz es necesario difundir entre la población los conceptos elementales del fenómeno tsunami, empleando para ello folletos de divulgación e incluyendo estos conceptos en los cursos regulares de la educación primaria. El conocimiento elemental más importante es que el terremoto produce al tsunami y que al sentir un terremoto se debe buscar de inmediato un sitio alto y seguro. Aún cuando no se produzca un tsunami, es mejor esperar un par de horas a que pase el peligro, que sufrir las consecuencias.

La situación de Tumaco demanda labores de mitigación prioritarias en lo referente a la adecuación de vías de evacuación (por Ej.: El puente El Pindo), a la construcción de infraestructura sismo-tsunami resistente para planteles educativos y centros de atención de salud y a la labor de preparación de las autoridades para que puedan actuar de manera acertada durante y después de un evento de esta indole.

\section{Agradecimientos}

La elaboración de este estudio fue posible gracias al financiamiento de la Dirección General Marítima de Colombia, COLCIENCIAS y al apoyo de la Agencia de Cooperación Internacional de Japón (JICA) en el marco de cooperación Sur-Sur-México-Japón en el programa de envío de expertos a terceros países, noviembre 1999.

\section{Referencias}

BECK, L., AND L. RUFF, 1984. The Rupture Process of the Great 1979 Colombia Earthquake: Evidence for the Asperity Model, J. Geophys. Res., 89, 9819291.

GOTO C., Y OGAWA, N. SHUTO, and F. IMAMURA, 1997. IUGG/IOC TIME Project: Numerical Method of Tsunami Simulation with the Leap-Frog Scheme, Intergovernmental Oceanographic Commission of UNESCO, Manuals and Guides \# 35, Paris, 4 Parts.

HERD, D. G., T. LESLIE, H. MEYER, J. L. ARANGO, W. J. PERSON, AND C. MENDOZA, 1981. The Great Tumaco, Colombia Earthquake of 12 December 1979, Science, 211, 4481, 441 - 445.

KANAMORI, H., and K. MCNALLY, 1982. Variable rupture mode of the subduction zone along the Ecuador - Colombia coast, Bull. Seismol. Soc. Am., 72, $1241-1253$.

KELLEHER, J., 1972. Rupture zones of large South American earthquakes and some predictions, J. Geophys. Res., 77, 2087 . 2103.

LAMB H., 1932. Hydrodynamics, 6th edition, 738, Cambridge Univ. Press.

MANSINHA, L. and E. SMYLIE, 1971. The Displacement Field of Inclined Faults, Bull. Seismol. Soc. Am. 61, 1433-1440.

ORTIZ M., E. GOMEZ-REYES, and H. VELEZ-MUÑOZ, 2000a. A Fast Preliminary Estimation Model for Transoceanic Tsunami Propagation. Geof. Int., Vol., 39, Num. 3, pp 1-14.

ORTIZ M., S.K. SINGH, V. KOSTOGLODOV and J. PACHECO, 2000b. Source areas of the Acapulco-San Marcos, Mexico earthquakes of 1962 ( $M 7.1 ; 7.0$ ), as constrained by tsunami and uplift records. Geof. Int., Vol., 39, Num. 4, pp 337 - 348.

ORTIZ M., V. KOSTOGLODOV, S.K. SINGH and J. PACHECO, 2000c. New constraints on the uplift of october 9, 1995 Jalisco - Colima earthquake (Mw 8 ) based on the analysis of tsunami records at manzanillo and Navidad, Mexico. Geof. Int., Vol., 39, Num. 4, pp 349 - 357.

PEDLOSKY, J., 1979. Geophysical Fluid Dynamics, 624 pp. SpringerVerlag.

RAMIREZ J., y J. GOBERNA, 1980. Informe Preliminar Terremotos Colombianos noviembre 23 y diciembre 12 de 1979. Reporte Interno. Instituto Geofísico de los Andes Colombianos (Universidad Javeriana)

SOLOVIEV, L. And N. GO, 1975. A catalogue of tsunamis on the eastern shore of the Pacific Ocean (1513-1968). Nauka Publishing House, Moscow, USSR, 204p. Can. Transl. Fish. Aquat. Sci. 5078, 1984.

SOLOVIEV, S, L., CH. GO, and KH.S KIM, 1992. Catalog of tsunamis in the Pacific 1969-1982. Academy of Sciences of the USSR Soviet Geophysical Committee. Results of Researches on the International Geophysical Projects, Moscow.

SHUTO, N., T. SUZUKI, K. HASEGAWA, and K. INAGAKI, 1986. A study of numerical techniques on the tsunami propagation and run up. Science of Tsunami Hazard, 4, 111 - 124.

SMITH, W. H. F. And D. T. SANDWELL, 1997. Global Seafloor Topography from Satellite Altimetry and Ship Depth Soundings, Science, 277, 1956 - 1962, 26 september.

TAKAHASHI, TO., TA. TAKAHASHI, N. SHUTO, F. IMAMURA, and $M$. ORTIZ, 1995. Source Models of the 1993 Hokkaido Nansei-Oki earthquake tsunami, A Topical Issue of Pure and Applied Geophysics, Vol. 144, No. 3/4, 1995. 


\section{Localidad \# 1 Localidad \# 2 Localidad \# 3 Localidad \# 4}

\begin{tabular}{|l|c|c|c|c|c|c|c|c|}
\hline Plano de Falla & T, min & H, m & T, min & H, m & T, min & H, m & T, min & H, m \\
\hline a & 63 & 0.2 & 60 & 0.1 & 64 & 0.1 & 48 & 0.3 \\
\hline B (1979) & 41 & 0.9 & 40 & 0.6 & 39 & 0.7 & 30 & 1.8 \\
\hline C & 55 & 0.2 & 60 & 0.1 & 59 & 0.1 & 41 & 0.4 \\
\hline d & 80 & 0.1 & 80 & 0.1 & 77 & 0.07 & 58 & 0.2 \\
\hline
\end{tabular}

Tabla 1

Alturas y tiempo de llegada del primer máximo del tsunami sobre distintos puntos del área urbana de Tumaco, para diferentes ubicaciones del plano de falla. T (tiempo, minutos); $\mathrm{H}$ (altura, metros)

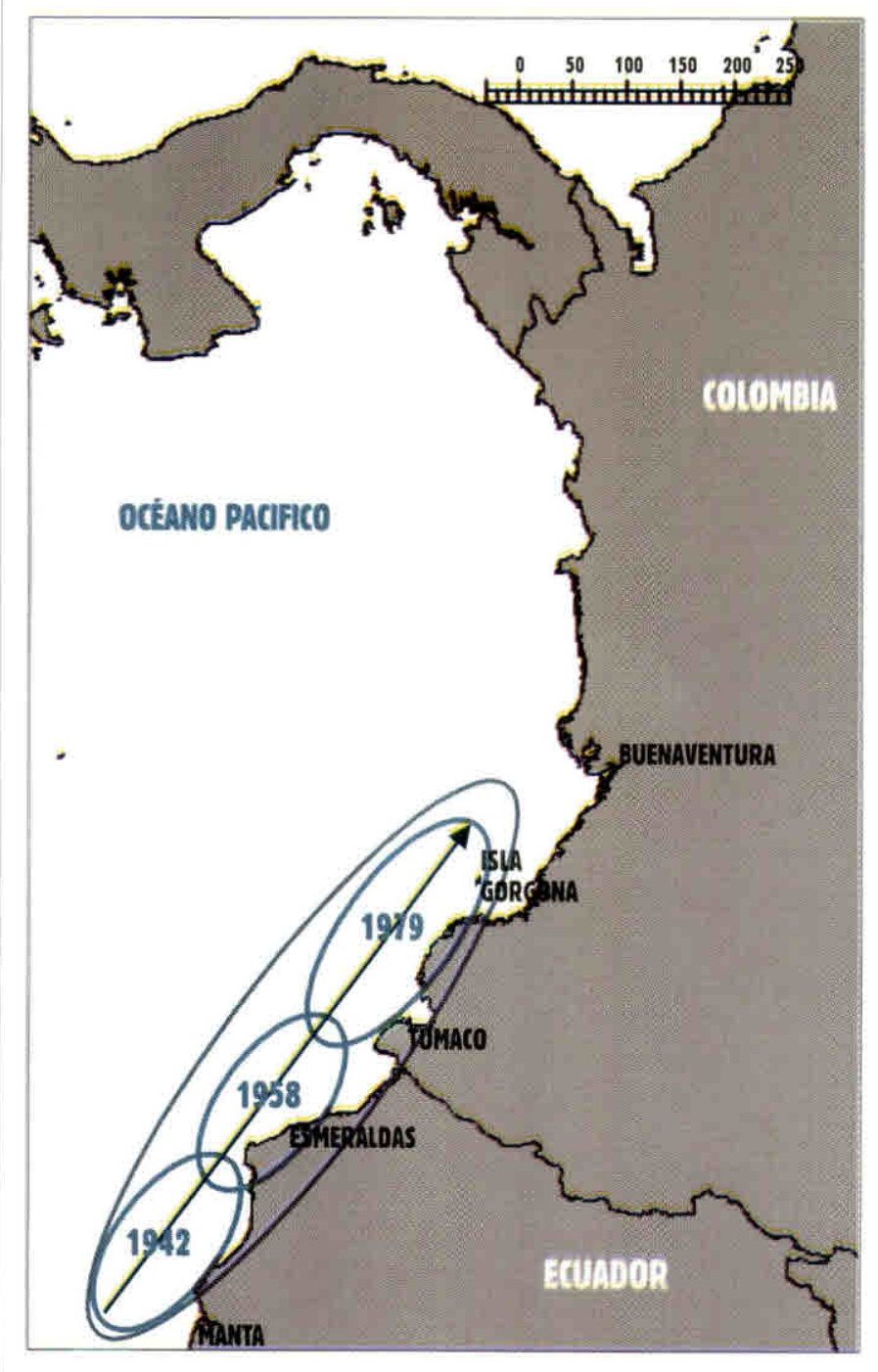

\section{Figura 1}

Se indica esquemáticamente la dirección y longitud de ruptura de los sismos de Ecuador-Colombia de 1906, 1942, 1958 y 1959. La localización de las áreas de ruptura correspondientes se puede consultar en: Kelleher, 1972; Herd et al., 1981; Beck and Ruff, 1984

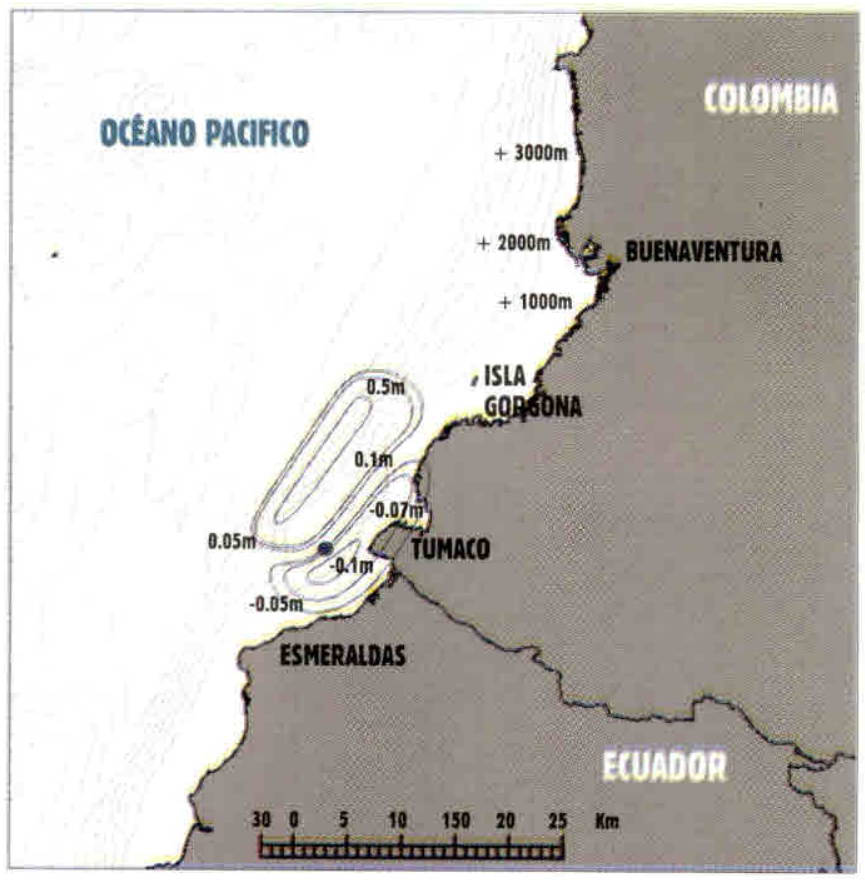

\section{Figura 2}

Deformación vertical cosismica del lecho marino calculada con el modelo de deformación de Manshina y Smilye, 1971. Los contornos de 5,50 y $100 \mathrm{~cm}$ indican la elevación cosísmica del lecho marino. La depresión cosísmica del lecho marino se indica con los contornos de $-5,-10$ y $-20 \mathrm{~cm}$. El círculo indica el epicentro del sismo del $12 \mathrm{de}$ diciembre de 1979 . El relieve del fondo marino se indica con las isóbatas de 1000,2000 y $3000 \mathrm{~m}$. 


\section{Figura 3}

Mareograma de Esmeraldas-Ecuador del 12 de diciembre de 1979.

Figura tomada de Soloviev et al., 1992. La recesión en el nivel del mar inicia minutos después del tiempo de origen del sismo (07:59:03 UT; 02:59:03 hora local).
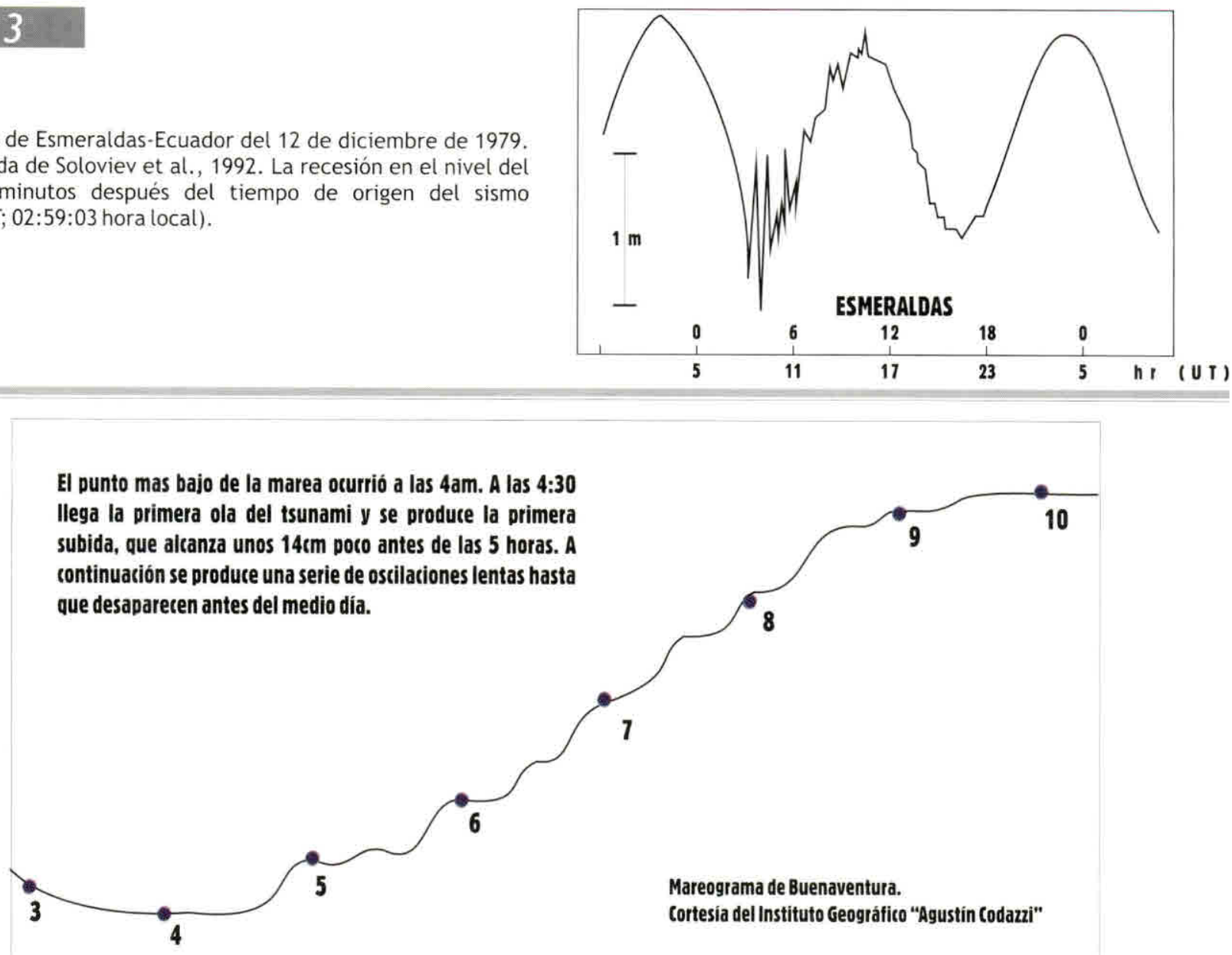

\section{Figura 4}

Mareograma de Buenaventura-Colombia del 12 de diciembre de 1979. Los números sobre el mareograma indican la hora local. La diferencia de nivel entre las 4 y las 10 horas es $1.88 \mathrm{~m}$.

Tiempo, horas (hora local)

\section{Figura 5}

Superior: segmento del mareograma de Esmeraldas del 12 de diciembre de 1979.

Inferior: mareograma sintético de Esmeraldas. El tiempo de origen del sismo corresponde a las 2:59:04 hora local. El eje de tiempo es el mismo para ambas figuras.
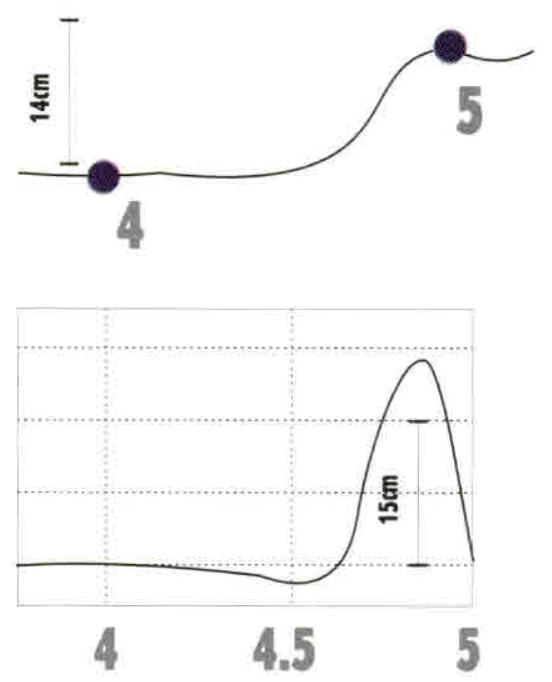

Tiempo, horas (hora local)

\section{Figura 6}

Superior: segmento del mareograma de Buenaventura del 12 de diciembre de 1979.

Inferior: mareograma sintético de Buenaventura. El tiempo de origen del sismo corresponde a las 2:59:04 hora local. El eje de 


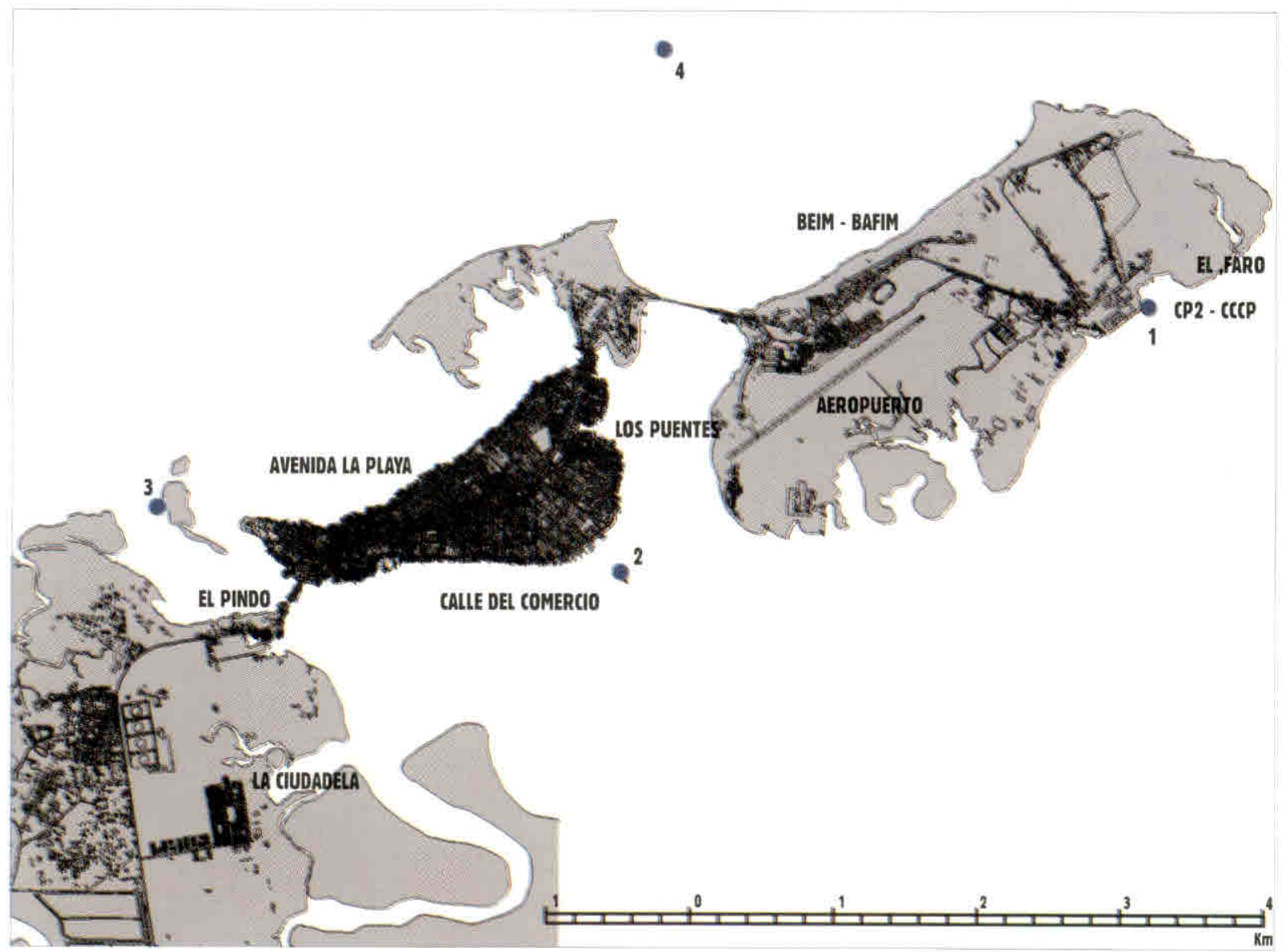

Figura 7

Municipio de Tumaco. Se indican los cuatro puntos seleccionados para el registro de los mareogramas sintéticos que se observan en la figura 8. Los sectores de la Avenida de La Playa, los Puentes y la Calle del Comercio sobre la Isla de Tumaco son los más densamente poblados.
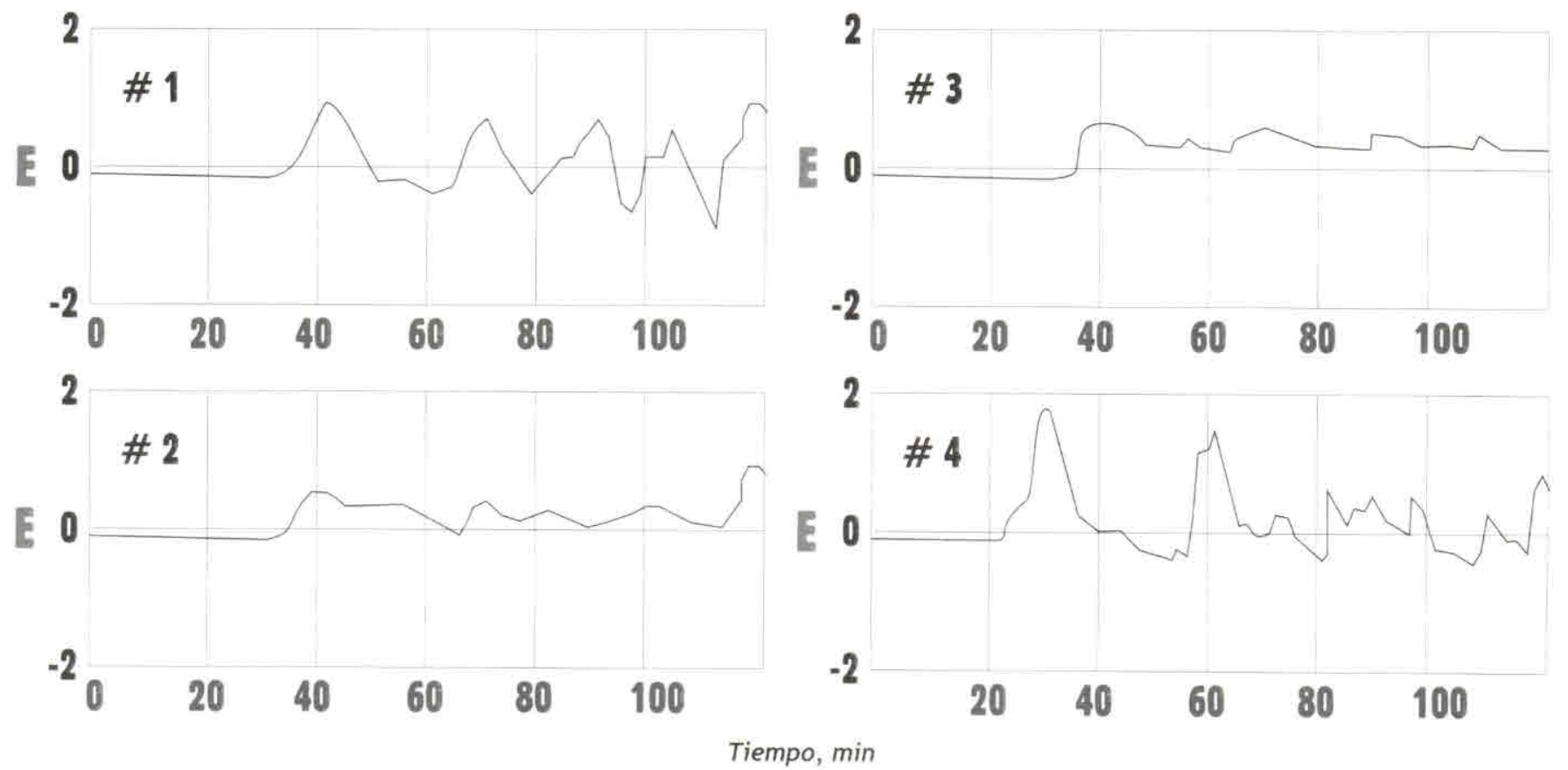


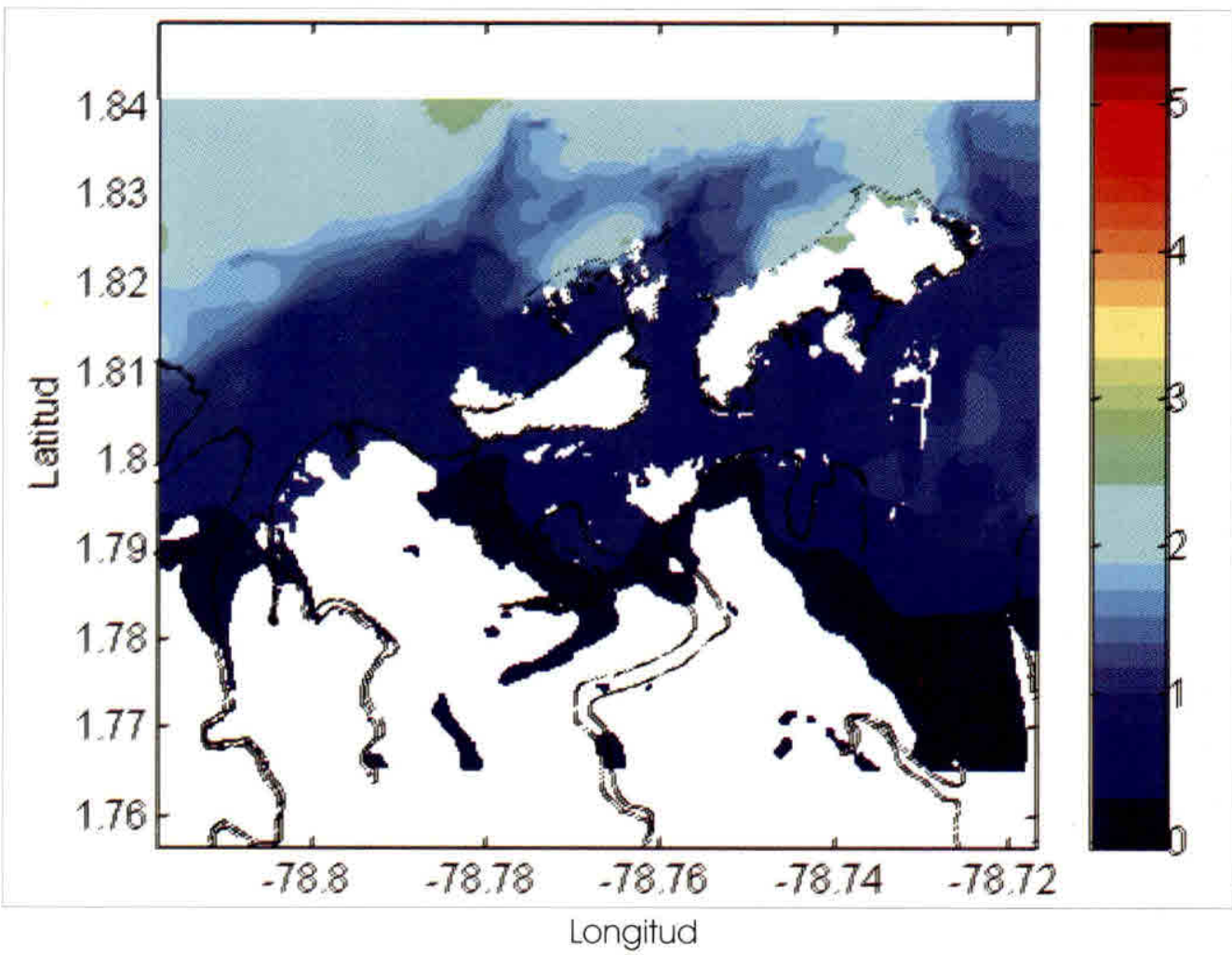

Resultados de altura y extensión horizontal de inundación en Tumaco en caso de un tsunami similar al de 1979. La escala de color indica la máxima altura (metros respecto al NMM) alcanzada por el tsunami. Las áreas sin color en las islas y el continente no alcanzan a cubrirse de agua durante el tsunami. La mayor parte de la superficie de la Isla La Viciosa queda cubierta por agua.

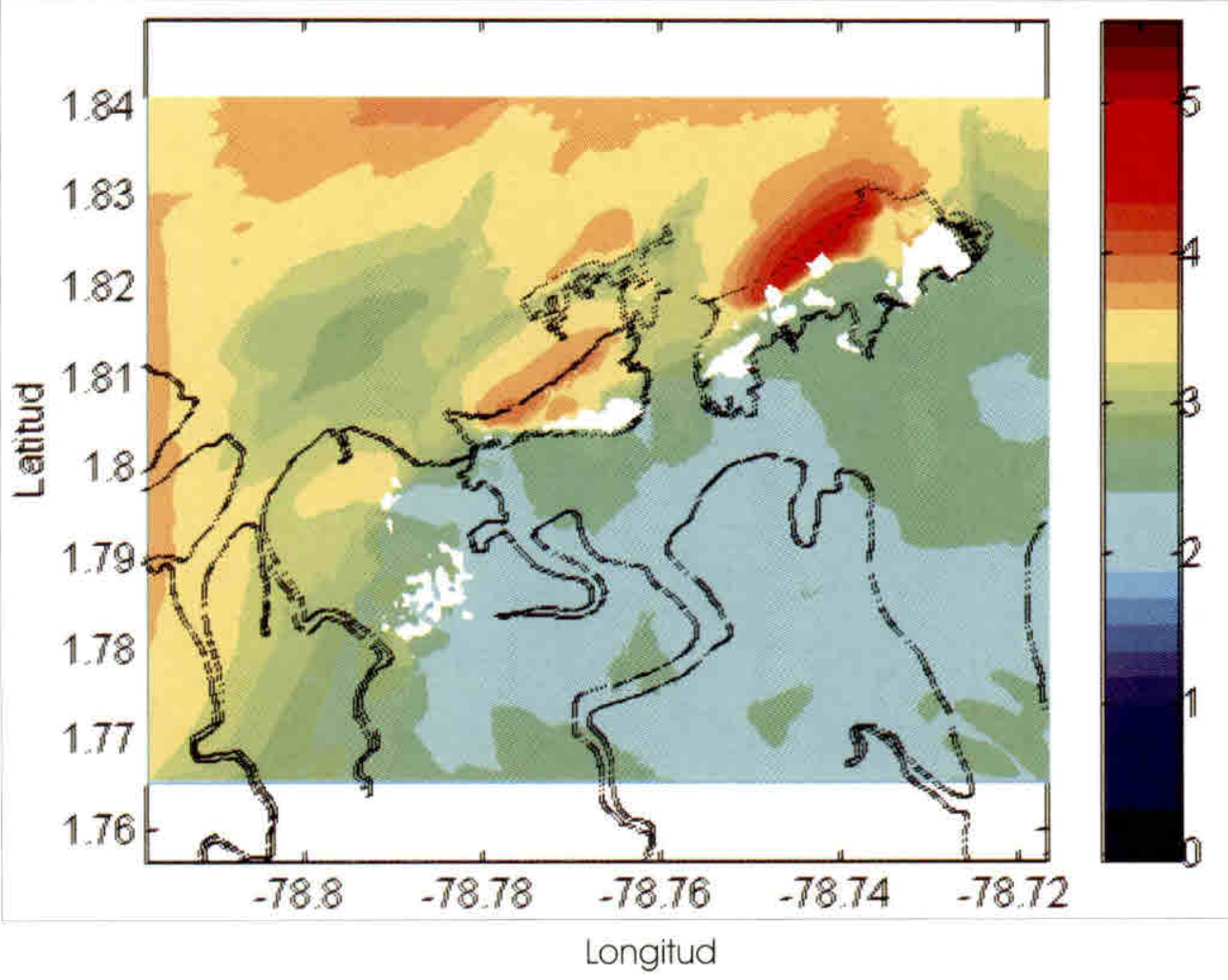

Resultados de altura y extensión horizontal de inundación en Tumaco en caso de que un tsunami similar al de 1979 


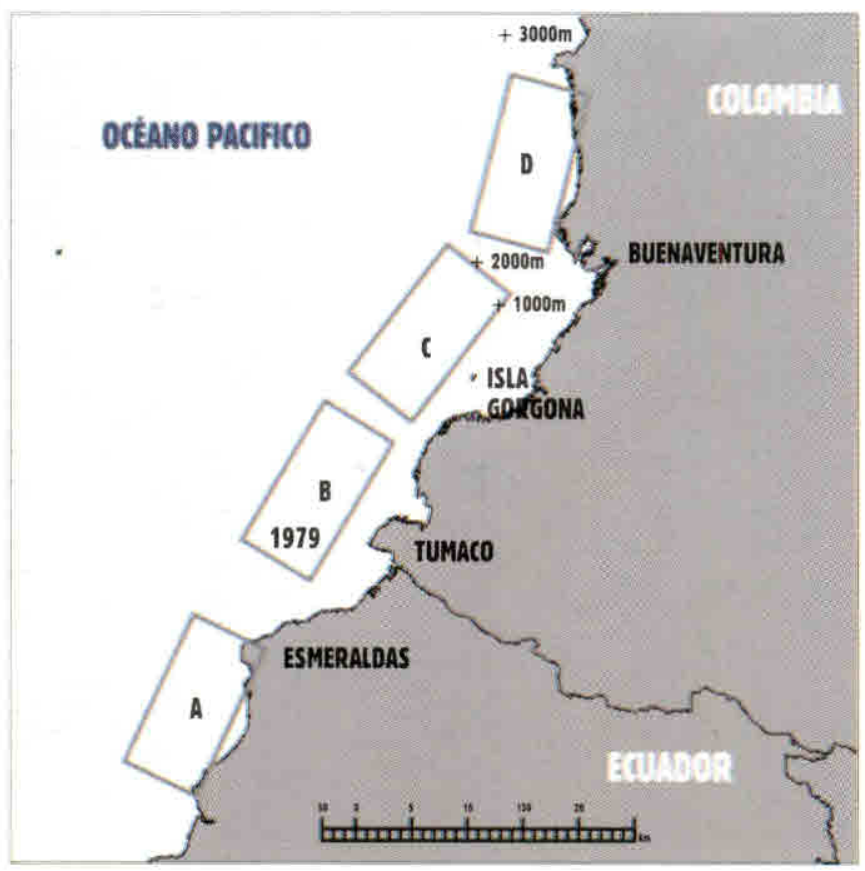

Figura 11

Los rectángulos a, b, c y d indican la posición de los planos de falla empleados para calcular la forma inicial del tsunami similar al tsunami del 12 de diciembre de 1979. El relieve del fondo marino se indica con las isóbatas de 1000,2000 y $3000 \mathrm{~m}$.

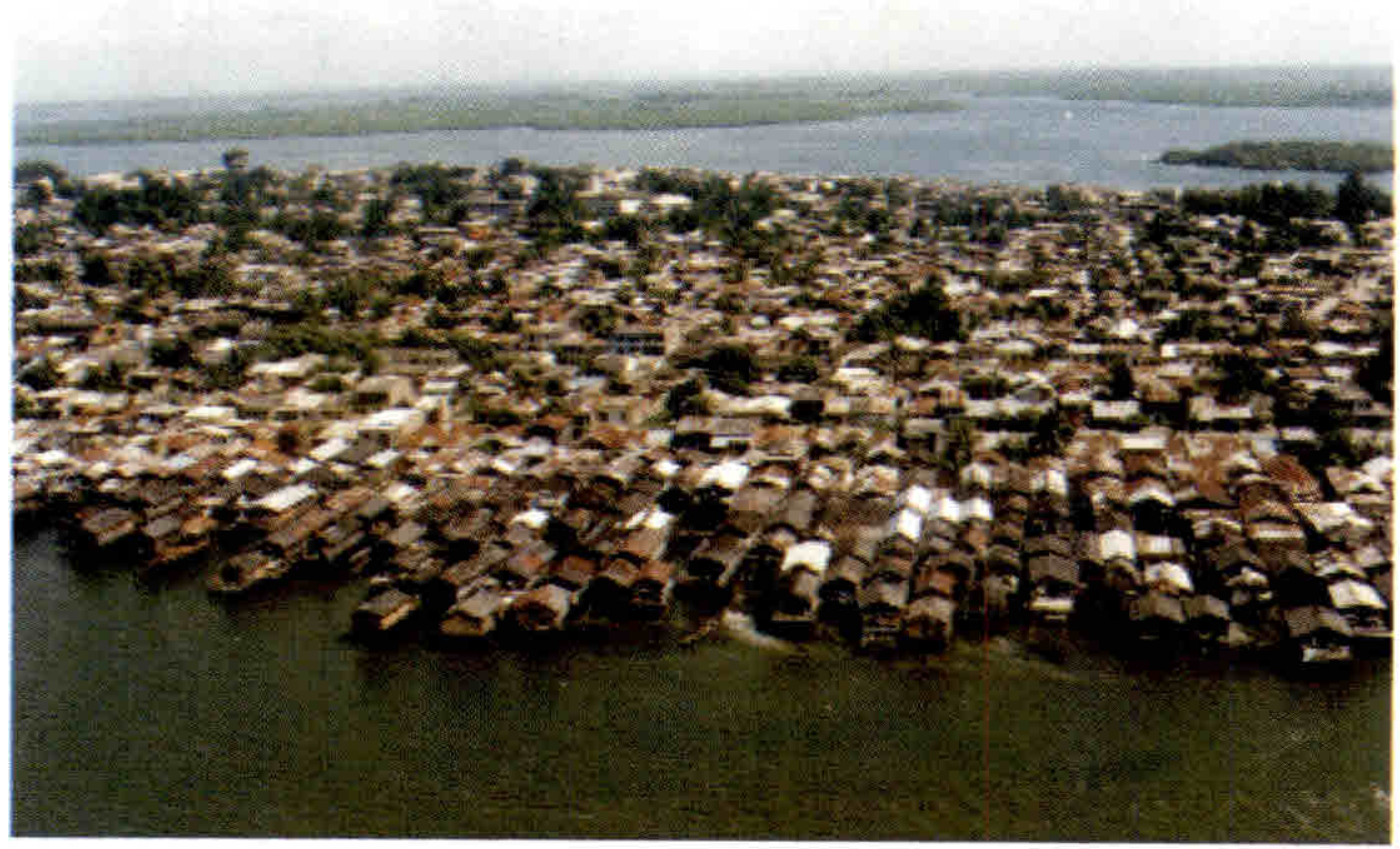




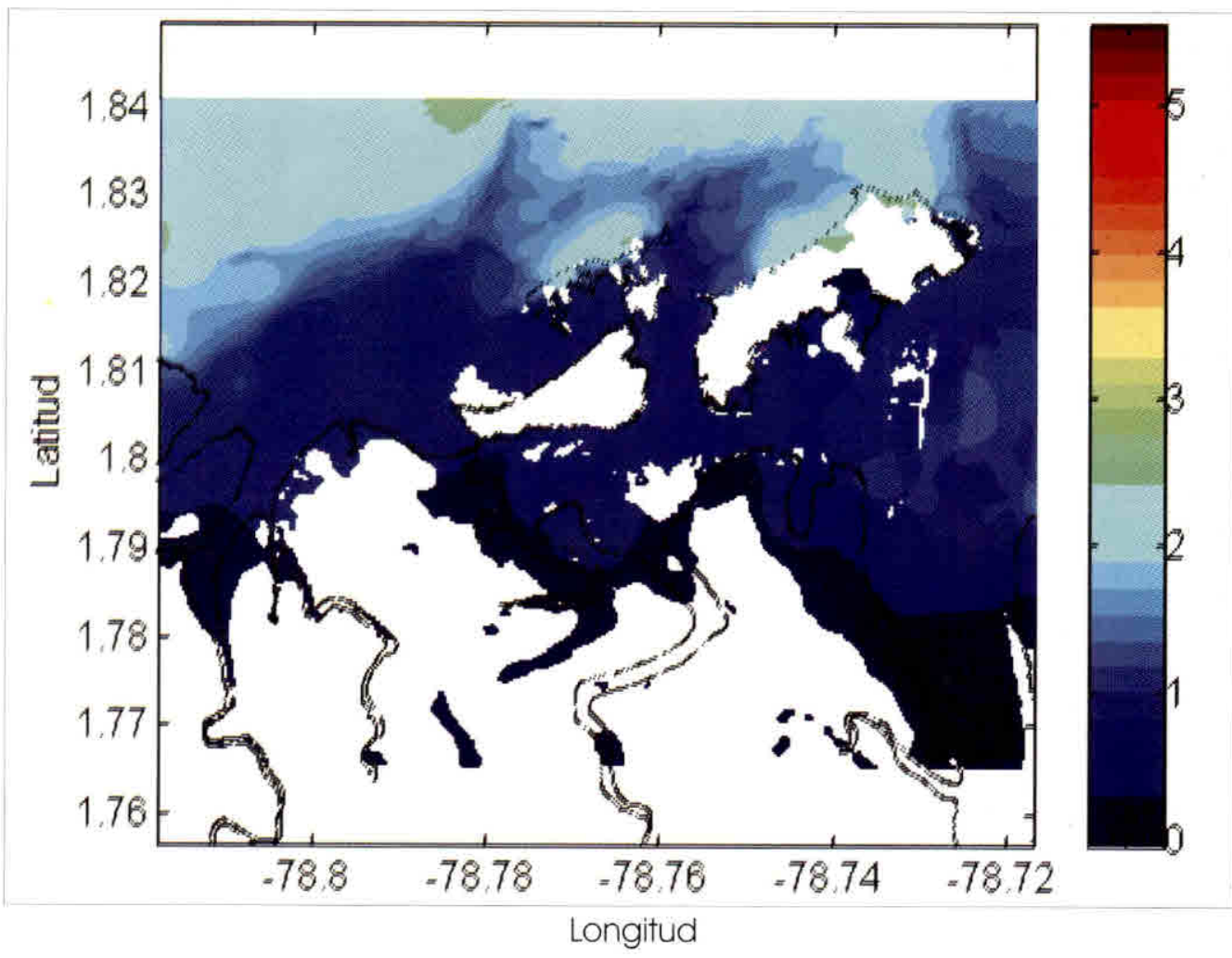

Resultados de altura y extensión horizontal de inundación en Tumaco en caso de un tsunami similar al de 1979. La escala de color indica la máxima altura (metros respecto al NMM) alcanzada por el tsunami. Las áreas sin color en las islas y el continente no alcanzan a cubrirse de agua durante el tsunami. La mayor parte de la superficie de la Isla La Viciosa queda cubierta por agua.

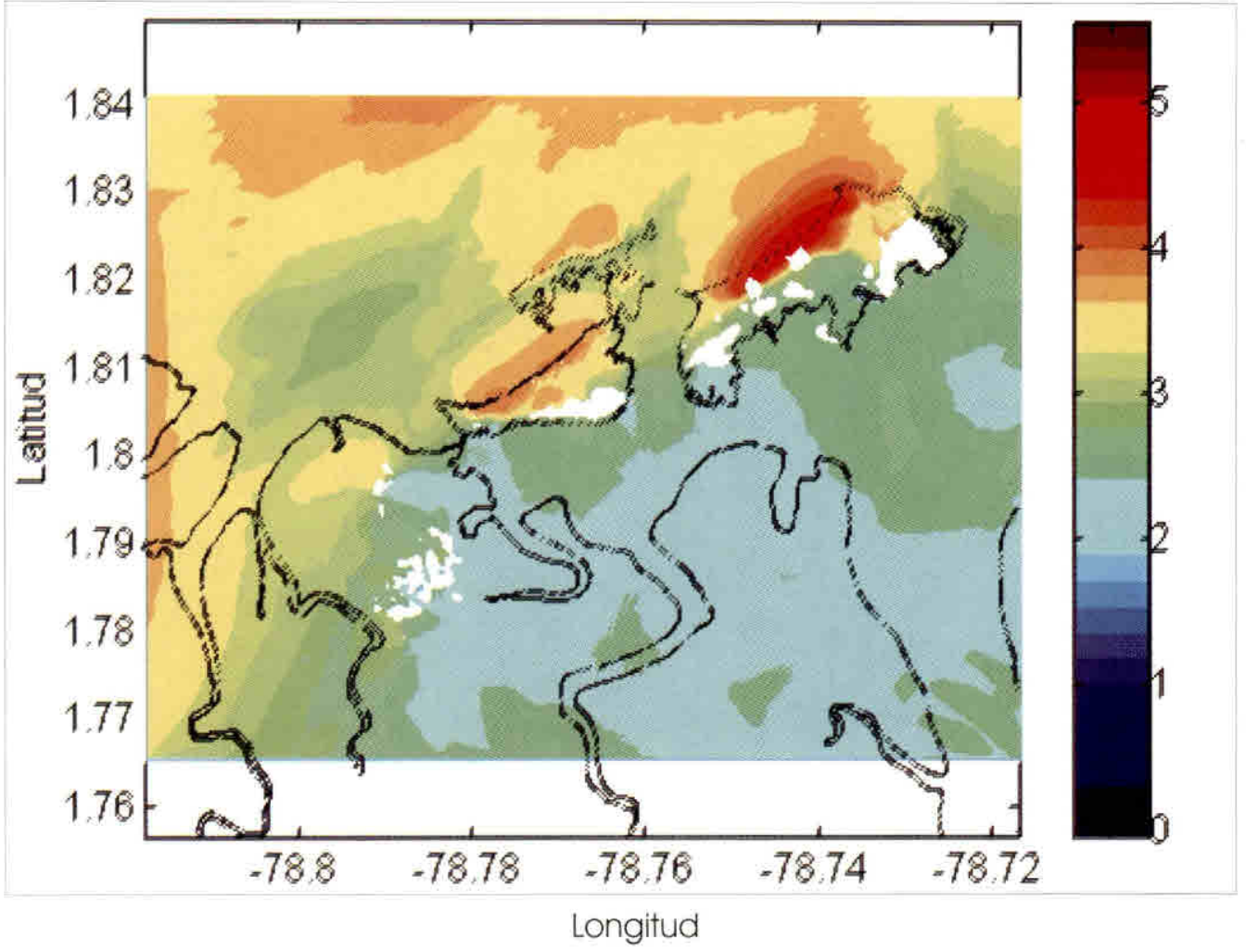

Resultados de altura y extensión horizontal de inundación en Tumaco en caso de que un tsunami similar al de 1979 hubiese ocurrido durante la marea alta $(+1,75 \mathrm{~m}$ sobre el nivel medio del mar) La escala de color indica la máxima altura (metros respecto al NMM) alcanzada por el tsunami. Las áreas sin color en las islas y el continente no alcanzan a cubrirse de agua durante el tsunami. 


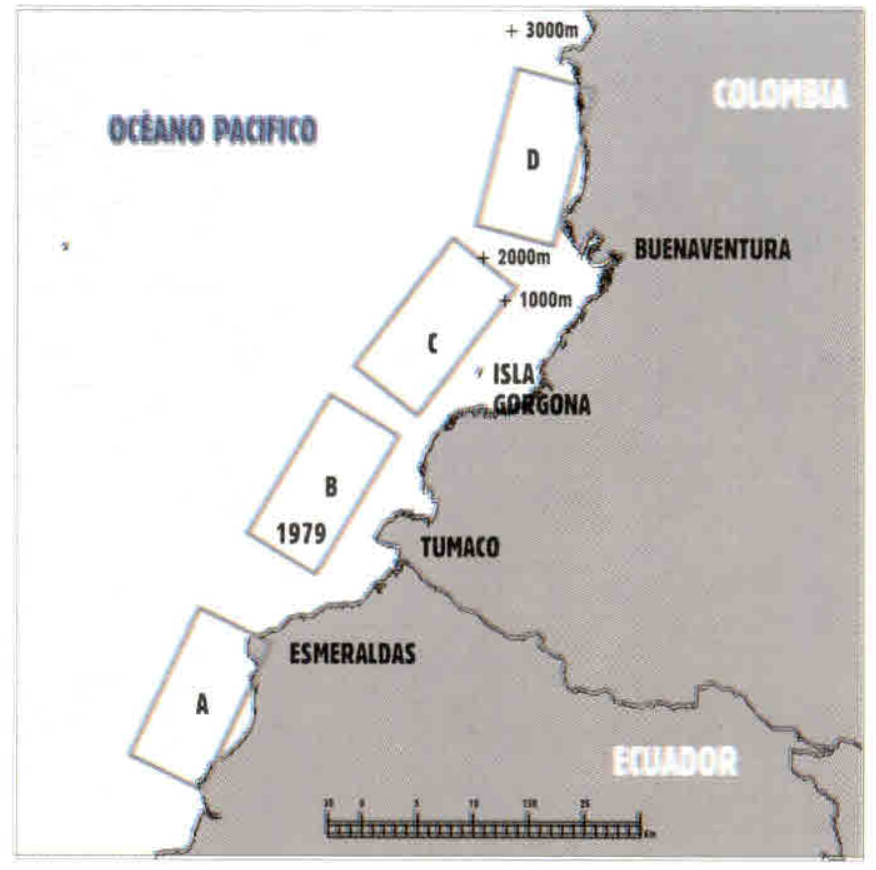

Figura 11

Los rectángulos a, b, c y d indican la posición de los planos de falla empleados para calcular la forma inicial del tsunami similar al tsunami del 12 de diciembre de 1979. El relieve del fondo marino se indica con las isóbatas de 1000,2000 y $3000 \mathrm{~m}$.

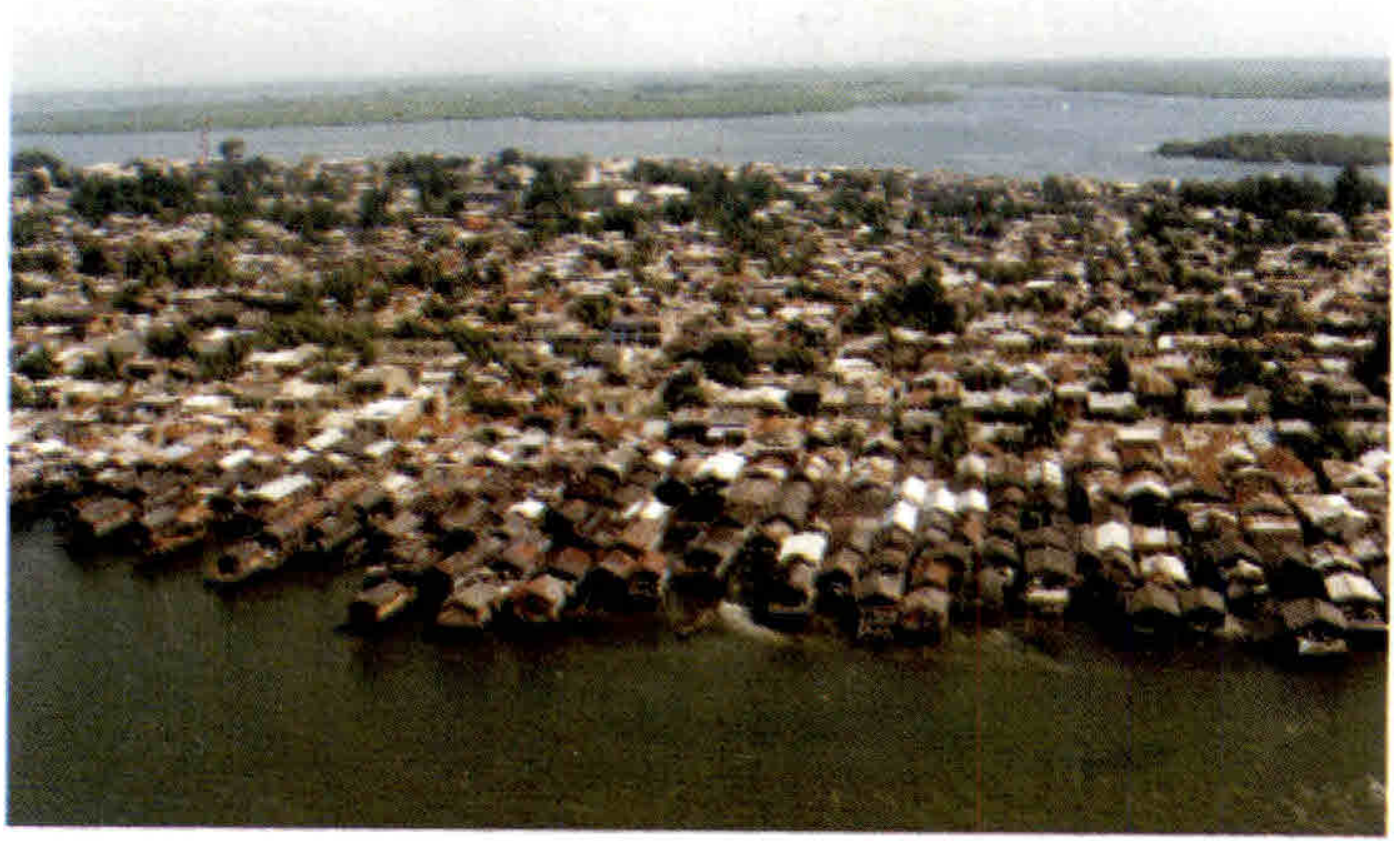

